\title{
Fundamental Equations of Nonlinear Fiber Optics
}

\author{
Haiqing Wei* and David V. Plant \\ Department of Electrical \& Computer Engineering \\ McGill University, Montreal, Canada H3A-2A7 \\ *hwei1@po-box.mcgill.ca
}

\begin{abstract}
A set of nonlinear differential equations are derived from the first principles, namely the Maxwell's equations and the material responses to electromagnetic excitations. The derivation retains the mathematical exactitude down to details. Still in compact and convenient forms, the final equations include the effect of group-velocity dispersion down to an arbitrary order, and take into account the frequency variations of the optical loss as well as the transverse modal function. Also established is a new formulation of multi-component nonlinear differential equations, which is especially suitable for the study of wide-band wavelength-division multiplexed systems of optical communications. The formulations are applied to discuss the problem of compensating the optical nonlinearity of fiber transmission lines using optical phase conjugation. Two system configurations are identified suitable for nonlinearity compensation. One setup is mirror-symmetric and the other translationally symmetric about the optical phase conjugator, both being in a scaled sense.
\end{abstract}

Keywords: nonlinear fiber optics, fundamental equations, compensation, scaled nonlinearity, scaled symmetry.

\section{INTRODUCTION}

Due to the material nonlinear effects, a fiber-optic transmission line is a nonlinear channel. In state-of-theart transmission systems, a single-mode optical fiber may carry tens even more wavelength channels. Each wavelength carries $10 \mathrm{~Gb} / \mathrm{s}$ or higher data rate, and the signal may travel several thousand of kilometers in the fiber. With such long transmission distance at such high data rate, the optical nonlinearity of the fiber becomes significant. Indeed, fiber nonlinearity has become one of the major limiting factors in practical transmission system. ${ }^{1,2}$ The theory of nonlinear guided-wave optics, in particular nonlinear fiber optics, has played and should continue to play an important role in understanding fiber-optic signal transmissions, the generation and propagation of ultra-fast laser pulses in optical fibers, and fiber Raman amplifiers, to name just a few. The available formulations in the literature often use a single-component representation of the optical signals, treat the effect of group-velocity dispersion (GVD) up to the dispersion slope, and usually neglect the variations of the optical loss, the transverse mode function etc. across the signal frequency band. ${ }^{3,4}$ Although these approximations simplify the equations and offer some convenience, they may have already been or will soon be broken down by the rapid growth of the signal bandwidth. Indeed, modern transmission systems have already been carrying several tens of wavelength-division multiplexed (WDM) channels across an optical bandwidth in excess of $30 \mathrm{~nm}$. Some pioneering systems have even operated with a bandwidth close to $100 \mathrm{~nm}$. With such a wide bandwidth and so many WDM channels, it is rather inconvenient to use a single-component equation to describe the dynamics of all signals, and the higher-order GVD effects start to play important roles in the dynamics of signal propagations, especially the nonlinear interaction among the signals. Recent advancements in fiber technologies have produced dispersion-compensating fibers (DCFs) that are capable of compensating simultaneously the dispersion and the slope of transmission fibers, leaving the residual dispersion dominated by the higher-order derivatives of GVD. ${ }^{5-10}$

Here we shall derive a set of nonlinear differential equations from the first principles, namely the Maxwell's equations and the material responses to electromagnetic excitations. The derivation retains the mathematical exactitude down to details. Each approximation is justified and the scope of its applicability is discussed. Still in compact and convenient forms, the final equations include the effect of GVD down to an arbitrary order, and take into account the frequency variations of the optical loss as well as the transverse modal function. Also established 
is a new formulation of multi-component nonlinear differential equations, in which the total electromagnetic field is represented as a sum of signals with different center frequencies. The multi-component formulation is especially suitable for the study of wide-band wavelength-division multiplexed systems of optical communications. We then apply the formulations to discuss the problem of compensating the optical nonlinearity of fiber transmission lines, either by themselves or by specialty fibers, with the help of (an) optical phase conjugation (conjugator) (OPC). Two system configurations with distinct symmetric properties are identified suitable for the compensation of fiber nonlinearity in optical transmission lines. In one setup, two fiber lines are arranged mirror-symmetric about the OPC to compensate each other's nonlinearity, while in the other setup, the two fiber lines are configured translationally symmetric about the OPC. In either case, the symmetry is in a scaled sense, and refers to the space variations of the signal power and the fiber parameters such as dispersion, dispersion slope, and nonlinear coefficients.

\section{DERIVATION OF THE NONLINEAR SCHRÖDINGER EQUATIONS}

This section should derive the nonlinear Schrödinger equations, which serve as the theoretical basis of nonlinear fiber optics. In dielectric optical waveguides, e.g. silica glass fibers, there is no source of electric charge, nor source of current, that is able to excite electromagnetic (EM) waves at the optical frequency. The magnetic response of most dielectrics is negligible at optical frequencies. The optics of dielectric waveguides is governed by Maxwell's equations, ${ }^{11}$

$$
\begin{aligned}
\nabla \times \mathbf{E} & =-\mu_{0} \frac{\partial \mathbf{H}}{\partial t}, \\
\nabla \times \mathbf{H} & =\epsilon_{0} \frac{\partial \mathbf{E}}{\partial t}+\frac{\partial \mathbf{P}}{\partial t}, \\
\nabla \cdot\left(\epsilon_{0} \mathbf{E}+\mathbf{P}\right) & =0, \\
\nabla \cdot \mathbf{H} & =0,
\end{aligned}
$$

and the material equation, ${ }^{3,4}$

$$
\begin{aligned}
\mathbf{P}(\mathbf{r}, t) & =\epsilon_{0} \int \chi^{(1)}(\mathbf{r}, s) \mathbf{E}(\mathbf{r}, t-s) d s \\
& +\epsilon_{0} \int \chi^{(3)}\left(\mathbf{r}, t_{1}, t_{2}, t_{3}\right) \vdots \mathbf{E}\left(\mathbf{r}, t-t_{1}\right) \mathbf{E}\left(\mathbf{r}, t-t_{2}\right) \mathbf{E}\left(\mathbf{r}, t-t_{3}\right) d t_{1} d t_{2} d t_{3},
\end{aligned}
$$

where $\chi^{(1)}$ and $\chi^{(3)}$ are the linear and the third-order nonlinear susceptibilities of the dielectric material respectively. Although Maxwell's equations describe the optical phenomena with the highest accuracy, they seem to be rather complicated when directly applied to optical waveguides, which usually consist of regions with different dielectric properties. Within each region, however, the material is often uniform and isotropic, so that the vector $\mathbf{P}$ is always proportional and parallel to $\mathbf{E}$, if neglecting the nonlinear polarization for the moment. Then equation (3) is reduced to $\nabla \cdot \mathbf{E}=0$. By applying $\nabla \times$ to (1), using (2) and the identity,

$$
\nabla \times \nabla \times \mathbf{E}=\nabla(\nabla \cdot \mathbf{E})-\nabla^{2} \mathbf{E}=-\nabla^{2} \mathbf{E}
$$

it is obtained,

$$
\nabla^{2} \mathbf{E}(\mathbf{r}, t)=\frac{1}{c^{2}} \frac{\partial^{2}}{\partial t^{2}} \mathbf{E}(\mathbf{r}, t)+\mu_{0} \frac{\partial^{2}}{\partial t^{2}} \mathbf{P}
$$

with $1 / c^{2}=\epsilon_{0} \mu_{0}$. Both $\chi^{(1)}$ and $\chi^{(3)}$ may be complex quantities. The real part of $\chi^{(1)}$ represents the positionand frequency-dependent dielectric constant, while the imaginary part reflects the optical loss or gain in the materials, which may be regarded as frequency-independent, as the total signal bandwidth $W$ is usually much smaller than the center optical frequency $\omega_{0}$ of the band. The imaginary part of $\chi^{(3)}$ represents the two-photon absorption effect, which is negligible in fibers of silica glass. The real part of $\chi^{(3)}$ comes from two contributions, $\operatorname{Re}\left[\chi^{(3)}\right]=\chi_{K}^{(3)}+\chi_{R}^{(3)}$, where $\chi_{K}^{(3)}$ is responsible for the optical Kerr effect, which may be regarded as an 
instantaneous one, while $\chi_{R}^{(3)}$ is tied to the Raman scattering effect, which is a time-delayed process. ${ }^{3,4}$ So the material equation (5) may be simplified as,

$$
\begin{aligned}
\mathbf{P}(\mathbf{r}, t)= & \epsilon_{0} \int \operatorname{Re}\left[\chi^{(1)}(\mathbf{r}, s)\right] \mathbf{E}(\mathbf{r}, t-s) d s+i \epsilon_{0} \operatorname{Im}\left[\chi^{(1)}(\mathbf{r})\right] \mathbf{E}(\mathbf{r}, t)+ \\
& \epsilon_{0} \chi_{K}^{(3)}(\mathbf{r}) \vdots \mathbf{E}(\mathbf{r}, t) \mathbf{E}(\mathbf{r}, t) \mathbf{E}(\mathbf{r}, t)+\epsilon_{0} \int \chi_{R}^{(3)}(\mathbf{r}, s) \vdots \mathbf{E}(\mathbf{r}, t-s) \mathbf{E}(\mathbf{r}, t-s) \mathbf{E}(\mathbf{r}, t) d s .
\end{aligned}
$$

Now the guided-wave optics is described fairly accurately by equations (7) and (8), in addition to the proper boundary conditions ${ }^{11}$ connecting the fields in different dielectric materials. But there is still too much complexity, due to the vectorial nature of the EM field and the involved boundary conditions. Fortunately, optical fibers are designed to guide a few discrete modes, even just one mode, and the difference in the dielectric constant is small between the core and the cladding, which makes the guided modes very close to linearly polarized. ${ }^{12}$ In most fibers, the optical birefringence is either vanishingly weak to avoid the effect of PMD, or sufficiently strong to render the fiber polarization maintaining. For mathematical simplicity, it is assumed that all signals are co-linearly polarized when entering an optical fiber, and coupled into one polarization eigen state when the fiber is polarization maintaining. In case the signals are not co-linearly polarized, the mathematical description should be slightly modified to deal with the complication. However, the same physics remains to govern the nonlinear signal propagation in optical fibers, and still valid are most of the conclusions in the present proposal. With the linear polarization representation $\mathbf{E}(\mathbf{r}, t)=E(\mathbf{r}, t) \mathbf{e}_{1}$, equations (7) and (8) are simplified and combined as,

$$
\begin{array}{r}
\nabla^{2} E-\frac{1}{c^{2}} \frac{\partial^{2} E}{\partial t^{2}}-\frac{1}{c^{2}} \frac{\partial^{2}}{\partial t^{2}} \int \operatorname{Re}\left[\chi^{(1)}(s)\right] E(t-s) d s=\frac{i}{c^{2}} \operatorname{Im}\left[\chi^{(1)}\right] \frac{\partial^{2}}{\partial t^{2}} E+ \\
\frac{1}{c^{2}} \chi_{K}^{(3)} \frac{\partial^{2}}{\partial t^{2}} E^{3}+\frac{1}{c^{2}} \frac{\partial^{2}}{\partial t^{2}} \int \chi_{R}^{(3)}(s) E^{2}(t-s) E(t) d s .
\end{array}
$$

When the total signal bandwidth $W$ is not much more than a few $\mathrm{THz}$, it satisfies the condition $W \ll \omega_{0}$, as $\omega_{0} \approx 200 \mathrm{THz}$. The frequency dependence of the transverse modal function may be neglected, so that a trial solution,

$$
E(\mathbf{r}, t)=\operatorname{Re}\left[F(x, y) A(z, t) \exp \left(i \beta_{0} z-i \omega_{0} t\right)\right]
$$

with $\beta_{0}$ being the optical propagation constant at $\omega_{0}$, may be substituted into (9) to derive a differential equation for the envelope function $A(z, t)$. Since the fast variation is absorbed by the factor $\exp \left(i \beta_{0} z-i \omega_{0} t\right)$, the signal envelope $A(z, t)$ is expected to be slow-varying in both $z$ and $t$. The transverse modal function $F(x, y)$ is determined by substituting $F(x, y) A\left(z, \omega-\omega_{0}\right) \exp \left(i \beta_{0} z\right)$ into the Fourier transform of (9) with the right side set to zero. $F(x, y)$ is found to solve the eigen-value equation,

$$
\left(\frac{\partial^{2}}{\partial x^{2}}+\frac{\partial^{2}}{\partial y^{2}}\right) F(x, y)+\frac{\omega^{2}}{c^{2}} F(x, y)+\frac{\omega^{2}}{c^{2}} \operatorname{Re}\left[\chi^{(1)}(x, y, \omega)\right] F(x, y)=\beta^{2}(\omega) F(x, y) .
$$

Note that the eigen-value $\beta^{2}$ is $\omega$-dependent, which may be expanded into Taylor series,

$$
\beta^{2}(\omega)=\beta_{0}^{2}+2 \beta_{0} \sum_{k=1}^{+\infty} \frac{\beta_{k}}{k !}\left(\omega-\omega_{0}\right)^{k}, \quad \text { with }\left.\beta_{k} \stackrel{\text { def }}{=} \frac{1}{2 \beta_{0}} \frac{d^{k} \beta^{2}(\omega)}{d \omega^{k}}\right|_{\omega=\omega_{0}} .
$$

When transformed back into the time domain, equation (11) leads to,

$$
\begin{array}{r}
\frac{\partial^{2} E}{\partial x^{2}}+\frac{\partial^{2} E}{\partial y^{2}}-\frac{1}{c^{2}} \frac{\partial^{2} E}{\partial t^{2}}-\frac{1}{c^{2}} \frac{\partial^{2}}{\partial t^{2}} \int \operatorname{Re}\left[\chi^{(1)}(s)\right] E(t-s) d s= \\
\beta_{0}^{2} E+2 \beta_{0} F(x, y) \exp \left(i \beta_{0} z-i \omega_{0} t\right) \mathrm{B} A(z, t),
\end{array}
$$

where $B$ is a differential operator defined as,

$$
\mathrm{B} \stackrel{\text { def }}{=} \sum_{k=1}^{+\infty} \frac{\beta_{k}}{k !}\left(i \frac{\partial}{\partial t}\right)^{k}
$$


Substituting (10) and (13) into equation (9) in full, and multiplying both sides by $F^{*}(x, y)$ then integrating over the transverse plane, it is obtained the nonlinear Schrödinger equation (NLSE), which governs the propagation dynamics of the signal envelope in optical fibers,

$$
\frac{\partial A}{\partial z}-i \mathrm{~B} A+\frac{\alpha}{2} A=i \gamma|A|^{2} A+i\left(g \otimes|A|^{2}\right) A,
$$

where

$$
\begin{aligned}
\alpha & \stackrel{\text { def }}{=} \frac{\omega_{0}^{2}}{\beta_{0} c^{2}} \int \operatorname{Im}\left[\chi^{(1)}(x, y)\right]|F(x, y)|^{2} d x d y, \\
\gamma & \stackrel{\text { def }}{=} \frac{3 \omega_{0}^{2}}{8 \beta_{0} c^{2}} \int \chi_{K}^{(3)}(x, y)|F(x, y)|^{4} d x d y, \\
g(s) & \stackrel{\text { def }}{=} \frac{\omega_{0}^{2}}{4 \beta_{0} c^{2}} \int \chi_{R}^{(3)}(x, y, s)|F(x, y)|^{4} d x d y,
\end{aligned}
$$

assuming that $F(x, y)$ is normalized, and $\otimes$ denotes the convolution operator such that,

$$
\left[g \otimes|A|^{2}\right](z, t) \stackrel{\text { def }}{=} \int g(s)|A(z, t-s)|^{2} d s .
$$

In deriving equation (15), nonlinear products out of the signal band around $\omega_{0}$, and the Raman term involving $A^{2}(z, t-s) A^{*}(z, t)$, are dropped because the corresponding nonlinear processes are prevented by large phasemismatches, the term $\partial^{2} A / \partial z^{2}$ is neglected in view of the slow-varying nature of $A(z, t)$ in $z$, also neglected are terms involving the time-derivatives of $A(z, t)$ multiplied by $\operatorname{Im}\left[\chi^{(1)}\right], \chi_{K}^{(3)}$, or $\chi_{R}^{(3)}$, hence the name slowvarying envelope approximation. A long transmission link may use fibers of different types. Sometimes the fiber parameters may vary along a single piece of waveguide. So the quantities $(\mathrm{B}, \alpha, \gamma, g)$ are $z$-dependent in general. However, the corresponding derivatives with respect to $z$ may be neglected, as the $z$-dependence is usually step-wise, or extremely slow if continuous. The NLSE reads,

$$
\frac{\partial A}{\partial z}-i \mathrm{~B}(z) A+\frac{\alpha(z)}{2} A=i \gamma(z)|A|^{2} A+i\left[g(z) \otimes|A|^{2}\right] A,
$$

in the general version. After a frame transformation $(z, t) \rightarrow\left(z, t+\int^{z} \beta_{1}(\zeta) d \zeta\right)$, the NLSE takes a simpler form in the retarded frame, ${ }^{4}$

$$
\frac{\partial A}{\partial z}-i \mathrm{D}(z) A+\frac{\alpha(z)}{2} A=i \gamma(z)|A|^{2} A+i\left[g(z) \otimes|A|^{2}\right] A
$$

where

$$
\mathrm{D}(z) \stackrel{\text { def }}{=} \sum_{k=2}^{+\infty} \frac{\beta_{k}(z)}{k !}\left(i \frac{\partial}{\partial t}\right)^{k} .
$$

When the bandwidth of the optical signals becomes too large, it may violate the $\omega$-independent assumptions for the transverse modal function $F(x, y)$ and the loss or gain coefficient $\operatorname{Im}\left[\chi^{(1)}\right]$. However, the huge bandwidth is usually shared by many WDM channels, each of which, labelled by $n \in \mathbf{Z}$, is narrow-band around its own center frequency $\omega_{n}$. Within each WDM channel, the transverse modal function $F_{n}(x, y)$ and the loss or gain coefficient $\operatorname{Im}\left[\chi_{n}^{(1)}\right]$, both subscripted by $n$, are regarded as frequency-independent and valued at $\omega_{n}$. So the trial solution would be,

$$
E(\mathbf{r}, t)=\operatorname{Re} \sum_{n} F_{n}(x, y, z) A_{n}(z, t) \exp \left[i \int^{z} p_{n 0}(\zeta) d \zeta-i \omega_{n} t\right]
$$

where $p_{n 0}(z) \stackrel{\text { def }}{=} \beta\left(z, \omega_{n}\right)$ is the optical propagation constant at frequency $\omega_{n}$ and position $z, A_{n}$ is naturally the slow-varying envelope of the $n$th channel. The transverse modes $F_{n}(x, y), n \in \mathbf{Z}$, are determined by the eigen-value equations,

$$
\left(\frac{\partial^{2}}{\partial x^{2}}+\frac{\partial^{2}}{\partial y^{2}}\right) F_{n}(x, y)+\frac{\omega_{n}^{2}}{c^{2}} F_{n}(x, y)+\frac{\omega_{n}^{2}}{c^{2}} \operatorname{Re}\left[\chi^{(1)}\left(x, y, \omega_{n}\right)\right] F_{n}(x, y)=\beta^{2}(\omega) F_{n}(x, y), \quad \forall n \in \mathbf{Z},
$$


whose time-domain equivalents are,

$$
\begin{aligned}
\frac{\partial^{2} E_{n}}{\partial x^{2}} & +\frac{\partial^{2} E_{n}}{\partial y^{2}}-\frac{1}{c^{2}} \frac{\partial^{2} E_{n}}{\partial t^{2}}-\frac{1}{c^{2}} \frac{\partial^{2}}{\partial t^{2}} \int \operatorname{Re}\left[\chi^{(1)}(s)\right] E_{n}(t-s) d s=p_{n 0}^{2} E_{n} \\
& +2 p_{n 0} F_{n}(x, y) \exp \left[i \int^{z} p_{n 0}(\zeta) d \zeta-i \omega_{n} t\right] \mathrm{B}_{n} A_{n}(z, t), \quad \forall n \in \mathbf{Z}
\end{aligned}
$$

where

$$
E_{n}(\mathbf{r}, t)=F_{n}(x, y, z) A_{n}(z, t) \exp \left[i \int^{z} p_{n 0}(\zeta) d \zeta-i \omega_{n} t\right], \quad \forall n \in \mathbf{Z}
$$

and

$$
\mathrm{B}_{n}(z) \stackrel{\text { def }}{=} \sum_{k=1}^{+\infty} \frac{p_{n k}(z)}{k !}\left(i \frac{\partial}{\partial t}\right)^{k}, \text { with }\left.p_{n k}(z) \stackrel{\text { def }}{=} \frac{1}{2 p_{n 0}(z)} \frac{\partial^{k} \beta^{2}(z, \omega)}{\partial \omega^{k}}\right|_{\omega=\omega_{n}}, \forall k \geq 1, \forall n \in \mathbf{Z} .
$$

Substituting (23) and (25) into (9), projecting the field into individual transverse modes $F_{n}(x, y), n \in \mathbf{Z}$, and similarly, dropping the nonlinear products suffering from large phase-mismatches, neglecting $\partial^{2} A / \partial z^{2}$ and the terms involving the time-derivatives of $A(z, t)$ multiplied by $\operatorname{Im}\left[\chi^{(1)}\right], \chi_{K}^{(3)}$, or $\chi_{R}^{(3)}$, disregarding the $z$-derivatives of the fiber parameters and $F_{n}, \forall n \in \mathbf{Z}$, a group of multi-component NLSEs is obtained,

$$
\begin{array}{r}
\frac{\partial A_{n}}{\partial z}-i \mathrm{~B}_{n}(z) A_{n}+\frac{\alpha_{n}(z)}{2} A_{n}=i \sum_{k} \sum_{l} \gamma_{k l n}(z) A_{k} A_{l} A_{m}^{*} \exp \left[i \theta_{k l n}(z)\right] \\
-\sum_{k} \sum_{l} g_{k l n}(z) A_{k} A_{l} A_{m}^{*} \exp \left[i \theta_{k l n}(z)\right], \quad \forall n \in \mathbf{Z},
\end{array}
$$

where $m$ is determined by the condition $\omega_{m}=\omega_{k}+\omega_{l}-\omega_{n}$, and,

$$
\begin{aligned}
\alpha_{n}(z) & \stackrel{\text { def }}{=} \frac{\omega_{n}^{2}}{p_{n 0}(z) c^{2}} \int \operatorname{Im}\left[\chi_{n}^{(1)}(\mathbf{r})\right]\left|F_{n}(x, y)\right|^{2} d x d y, \\
\gamma_{k l n}(z) & \stackrel{\text { def }}{=} \frac{\omega_{n}^{2}}{8 p_{n 0}(z) c^{2}} \int\left[3 \chi_{K}^{(3)}(\mathbf{r})+2 G_{r}\left(\mathbf{r}, \omega_{n}-\omega_{k}\right)\right] F_{k} F_{l} F_{m}^{*} F_{n}^{*} d x d y, \\
g_{k l n}(z) & \stackrel{\text { def }}{=} \frac{\omega_{n}^{2}}{4 p_{n 0}(z) c^{2}} \int G_{i}\left(\mathbf{r}, \omega_{n}-\omega_{k}\right) F_{k} F_{l} F_{m}^{*} F_{n}^{*} d x d y, \\
G_{r}(\mathbf{r}, \omega)+i G_{i}(\mathbf{r}, \omega) & \stackrel{\text { def }}{=} \int \chi_{R}^{(3)}(\mathbf{r}, t) \exp (\omega s) d t, \text { both } G_{r} \text { and } G_{i} \text { are real valued, } \\
\theta_{k l n}(z) & \stackrel{\text { def }}{=} \int^{z}\left[p_{k 0}(\zeta)+p_{l 0}(\zeta)-p_{m 0}(\zeta)-p_{n 0}(\zeta)\right] d \zeta .
\end{aligned}
$$

As the Fourier transform of $\chi_{R}^{(3)}(t), G_{r}(\omega)+i G_{i}(\omega)$ is basically the Raman gain spectrum. Because $\chi_{R}^{(3)}$ is real valued, $G_{r}$ and $G_{i}$ are even and odd functions of $\omega$ respectively, namely, $G_{r}(-\omega)=G_{r}(\omega), G_{i}(-\omega)=-G_{i}(\omega)$. By a change of variables $(z, t) \rightarrow\left(z, t+\int^{z} p_{01}(\zeta) d \zeta\right)$, (28) may be rewritten as,

$$
\begin{array}{r}
\frac{\partial A_{n}}{\partial z}-i \mathrm{D}_{n}(z) A_{n}+\frac{\alpha_{n}(z)}{2} A_{n}=i \sum_{k} \sum_{l} \gamma_{k l n}(z) A_{k} A_{l} A_{m}^{*} \exp \left[i \theta_{k l n}(z)\right] \\
-\sum_{k} \sum_{l} g_{k l n}(z) A_{k} A_{l} A_{m}^{*} \exp \left[i \theta_{k l n}(z)\right], \quad \forall n \in \mathbf{Z},
\end{array}
$$

with

$$
\mathrm{D}_{n}(z) \stackrel{\text { def }}{=} \sum_{k=1}^{+\infty} \frac{p_{n k}(z)}{k !}\left(i \frac{\partial}{\partial t}\right)^{k}-p_{01}(z)\left(i \frac{\partial}{\partial t}\right), \quad \forall n \in \mathbf{Z} .
$$

After some tedious mathematical derivations, it is rather satisfying to see that the complicated phenomena of group-velocity dispersion and nonlinear interactions among optical signals are fully captured by the NLSEs 
(21) and (34) in their simple and appealing forms. Nevertheless, it is beneficial to highlight the theoretical model and analytical tools by recapping the assumptions and mathematical approximations being made in the above derivations. Such highlight should help to identify the applicability of the NLSEs as well as their limitations.

\section{Assumptions}

1. First of all, the optical field is believed to obey Maxwell's equations $(1-4)$.

2. The waveguide material is assumed to display third-order nonlinearity, with the electrical response given by $(5)$.

3. The waveguide material is step-wise uniform and isotropic. Namely, the waveguide consists of domains of uniform and isotropic materials, such that the polarization $\mathbf{P}$ (linear response) is always a scalar constant times the electrical field $\mathbf{E}$ within each domain.

4. The third-order nonlinearity consists of an instantaneous (Kerr) response and a time-delayed (Raman) scattering effect.

5. All signals are assumed co-linearly polarized when entering an optical fiber, and coupled into one polarization eigen state when the fiber is polarization maintaining.

6. The fiber parameters may be $z$-dependent, but their derivatives with respect to $z$ are always negligible.

These assumptions are responsible for distilling the first-principle equations $(1-4)$ and the material property (5) into the single scalar equation (9). Then the following approximations have been made in order to derive the single-component NLSE (21):

\section{Approximations for the single-component NLSE}

1. The optical loss or gain in the materials is regarded as frequency-independent.

2. The two-photon absorption effect is neglected in fibers of silica glass.

3. The frequency dependence is neglected for the transverse modal function.

4. The guided modes are treated as linearly polarized.

5. Nonlinear products out of the signal band are neglected.

6. The Raman term involving $A^{2}(z, t-s) A^{*}(z, t)$ is dropped because of a large phase-mismatch.

7. The term $\partial^{2} A / \partial z^{2}$ is neglected in view of the slow-varying nature of $A(z, t)$ in $z$.

8. Also neglected are terms involving the time-derivatives of $A(z, t)$ multiplied by $\operatorname{Im}\left[\chi^{(1)}\right], \chi_{K}^{(3)}$, or $\chi_{R}^{(3)}$.

The above approximations 1 and 3 are good when the total optical bandwidth $W$ is less than or on the order of a few THz. For systems with an optical bandwidth well exceeding the THz bandwidth limit, a channelized representation (23) may be used for the optical signals, and the following approximations may be made to derive a multi-component NLSE (34):

\section{Approximations for the multi-component NLSE}

1. The optical loss or gain in the materials may vary for different channels, however they are treated as frequency-independent within each channel.

2. The two-photon absorption effect is again neglected in fibers of silica glass.

3. The transverse modal function may depend on the center frequency of the channels, however no frequency dependence is considered within each channel. 
4. The guided modes are treated again as linearly polarized.

5. Nonlinear products out of the total signal band are neglected.

6. The Raman terms involving $A_{m}^{2}(z, t-s) A_{n}^{*}(z, t), \forall m, n \in \mathbf{Z}$ are dropped due to large phase-mismatches.

7. The terms $\partial^{2} A_{n} / \partial z^{2}, \forall n \in \mathbf{Z}$, are neglected in view of the slow-varying nature of $A_{n}(z, t)$ in $z$.

8. Also neglected are terms involving the time-derivatives of $A_{n}(z, t), \forall n \in \mathbf{Z}$, multiplied by $\operatorname{Im}\left[\chi^{(1)}\right], \chi_{K}^{(3)}$, or $\chi_{R}^{(3)}$.

\section{APPLICATIONS: NONLINEARITY COMPENSATION USING OPC}

It is known before that the nonlinearity of one fiber line may be compensated by that of another with the help of optical phase conjugation (OPC). However, all previous proposals and demonstrations ${ }^{13-17}$ work partially in fighting the fiber nonlinearity. They either are specialized to only one aspect of the nonlinear effects, or fail to work in the presence of dispersion slope or higher order dispersion effects. In, ${ }^{13}$ it is proposed that OPC may be employed in the middle of a long transmission line, not only to compensate the fiber dispersion, but also to cancel the integrated SPM in the two parts of the transmission line. Then, ${ }^{14}$ among others, demonstrates experimentally the compensation of dispersion and integrated SPM using OPC. However, the fiber nonlinearity is distributive in nature, due to the interplay between the fiber dispersion and the Kerr nonlinearity. Cancelling the integrated SPM merely compensates the single-channel nonlinearity in the sense of path average. The removal of such path-averaged nonlinearity represents a very limited improvement. In reference, ${ }^{15}$ a distributive nonlinear compensator is proposed using a specially designed fiber consisting of many segments. The method achieves better suppression to the SPM impairments, but it takes no account of the effect of higher-order dispersions, in particular the dispersion slope. Consequently, the method would not work with wide-band systems, e.g. highcapacity WDM systems and optical time-division multiplexed (OTDM) systems at very high-speed. Moreover, the distributive nonlinear compensator is difficult to fabricate, as it requires many fiber segments spliced together to approximate the distributive dispersion and nonlinearity in the transmission fiber. Reference ${ }^{16}$ suggests to compensate the SRS effect using spectral inversion, but again, up to the path average. The distributive nature of fiber nonlinearity defies once more such scheme of path-averaged compensation. Indeed, the proposed method of SRS compensation is severely limited by the pulse walk-off under an asymmetric profile of signal power about the spectral inverter. ${ }^{18}$ Finally, the experimental demonstration reported in $^{17}$ achieves probably the best results that represent the state-of-the-art. The experiment tries to make a fiber line symmetric about the point of OPC. In particular, it uses backward Raman pumping to approximate a symmetric power profile. The paper reported evidences of suppressed FWM, SPM, and XPM individually, and with a narrow system bandwidth. However, the Raman pumped fiber is the same as the transmission fiber, which is often too long for the Raman pump to maintain a constant gain. In other words, it is difficult to achieve a symmetric power profile. Furthermore, higher-order dispersions are not compensated by OPC, which could turn into a significant limitation in wide-band systems.

Using the mathematical formulations established above, we shall discuss nonlinearity compensation using two types of fiber arrangements with respect to the OPC, as shown in Fig.1. In one type of arrangement, the fiber parameters and the signal intensity are in scaled mirror symmetry about the OPC. While the other type is characterized by scaled translational symmetry. We argue that not only the dispersion, but also the slope and even higher-order dispersions should be carefully chosen, in order for one fiber to compensate the nonlinearity of the other, across a wide optical bandwidth containing many WDM channels. We also emphasize the important notions of scaled nonlinearity and scaled symmetry. Scaled nonlinearities and symmetries enable two fibers with a wide range of parameters to compensate each other's nonlinearity, as long as their parameters satisfy a set of proportional rules. In particular, a short piece of specialty fiber with very high dispersion may be used to compensate both the dispersion and the nonlinearity of a long transmission fiber. The dispersion, dispersion slope or higher order-dispersions are set in proportion to the parameters of the transmission fiber, and the specialty fiber may be erbium-doped or Raman pumped to have a gain proportional to the loss of the transmission fiber. In a mirror-symmetric setup, such specialty fibers may perfectly linearize fiber transmission lines. Besides practical applications in fiber transmission systems, the method of scaling nonlinearity and nonlinear compensation has a 
deep implication to a fundamental question in information theory. That is, fiber nonlinearity does not necessarily impose a limit to the channel capacity. In practice, there could be errors in scaling the fiber parameters, so that the nonlinear compensation may not be perfect. Nevertheless, excellent performance would still be achieved by choosing the parameters carefully according to the scaling rules. The possibility of nonlinearity compensation between two translationally symmetric fiber lines is the first of such discovery as far as we are aware. It is also a rather interesting scheme, in that the two fibers do not have to have opposite gain/loss coefficients: a lossy waveguide can be used to compensate a lossy transmission fiber, or the two fibers can be both amplifying. Although the translational setup is only capable of compensating weak nonlinearity up to the first-order perturbation, it may find wide applications in practical transmission systems, especially long-distance ones, where the nonlinearity of each fiber span is indeed only a perturbation, but the accumulation of nonlinearity along the distance can significantly distort the signals.
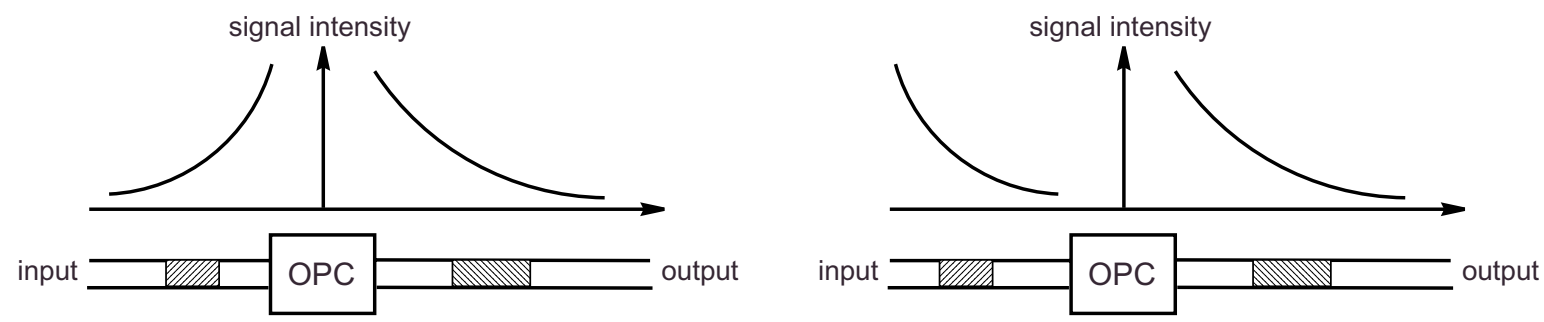

Figure 1. Two types of fiber arrangements for nonlinearity compensation with OPC: mirror-symmetric (left) and translationally symmetric (right).

A mirror-symmetric link may consist of a fiber line on the left stretching from $z=-L / R$ to $z=0, L>0$, $R>0$, followed by an OPC, then a fiber line on the right stretching from $z=0$ to $z=L$. The two fiber lines may carry wavelength-division multiplexed (WDM) signals $\sum_{n} A_{n}(z, t) \exp \left[i \int^{z} \beta\left(\zeta, \omega_{n}\right) d \zeta-i \omega_{n} t\right]$ and $\sum_{n} A_{n}^{\prime}(z, t) \exp \left[i \int^{z} \beta^{\prime}\left(\zeta, \omega_{n}^{\prime}\right) d \zeta-i \omega_{n}^{\prime} t\right]$ respectively, where $\forall n \in \mathbf{Z}, \omega_{n}$ and $\omega_{n}^{\prime}$ are the center frequencies of the WDM channels, $\omega_{0}$ is not necessarily equal to $\omega_{0}^{\prime}$, but $\omega_{n}-\omega_{0}=\omega_{0}^{\prime}-\omega_{-n}^{\prime}, \forall n \in \mathbf{Z}, A_{n}$ and $A_{n}^{\prime}$ are the slow-varying envelopes, while $\beta(z, \omega)$ and $\beta^{\prime}(z, \omega)$ are the $z$-dependent propagation constants. Being neglected is the frequency dependence of the transverse modal function. For mathematical simplicity, all optical signals are assumed co-linearly polarized in the fibers, in which the random PMD effect is always negligible. The dynamics of signal propagation in the two fiber lines is governed by two groups of coupled partial differential equations respectively,

$$
\begin{aligned}
\frac{\partial A_{n}}{\partial z}-i \mathrm{D}_{n}(z) A_{n}+ & \frac{\alpha_{n}(z)}{2} A_{n}=i \sum_{k} \sum_{l} \gamma_{k l n}(z) A_{k} A_{l} A_{m}^{*} \exp \left[i \theta_{k l n}(z)\right] \\
& -\sum_{k} \sum_{l} g_{k l n}(z) A_{k} A_{l} A_{m}^{*} \exp \left[i \theta_{k l n}(z)\right], \quad \forall n \in \mathbf{Z}, \\
\frac{\partial A_{n}^{\prime}}{\partial z}-i \mathrm{D}_{n}^{\prime}(z) A_{n}^{\prime}+ & \frac{\alpha_{n}^{\prime}(z)}{2} A_{n}^{\prime}=i \sum_{k} \sum_{l} \gamma_{k l n}^{\prime}(z) A_{k}^{\prime} A_{l}^{\prime} A_{m}^{\prime *} \exp \left[i \theta_{k l n}^{\prime}(z)\right] \\
& -\sum_{k} \sum_{l} g_{k l n}^{\prime}(z) A_{k}^{\prime} A_{l}^{\prime} A_{m}^{\prime *} \exp \left[i \theta_{k l n}(z)\right], \quad \forall n \in \mathbf{Z},
\end{aligned}
$$

$\forall n \in \mathbf{Z}$, where for the first fiber line, $\alpha_{n}$ is the attenuation coefficient around $\omega_{n}, \gamma_{k l n}$ is the Kerr nonlinear coefficient, $g_{k l n}$ is the Raman coupling coefficient among the channels, $\theta_{k l n}(z) \stackrel{\text { def }}{=} \int^{z}\left[\beta\left(\zeta, \omega_{k}\right)+\beta\left(\zeta, \omega_{l}\right)-\right.$ $\left.\beta\left(\zeta, \omega_{m}\right)-\beta\left(\zeta, \omega_{n}\right)\right] d \zeta$ is the phase-mismatch among the mixing waves, $m$ is determined by the condition $\omega_{m}=$ $\omega_{k}+\omega_{l}-\omega_{n}$, and the functional operator $\mathrm{D}_{n}$ is defined as in (35). The parameters $\alpha_{n}^{\prime}, \gamma_{k l n}^{\prime}, g_{k l n}^{\prime}, \theta_{k l n}^{\prime}$ and the operators $\mathrm{D}_{n}^{\prime}(z)$ are similarly defined for the second fiber line. It is an easy exercise to show that the complex conjugate of (36) reduce to (37), when the parameters satisfy the following rules of correspondence,

$$
\begin{aligned}
p_{n 1}(-z)-p_{01}(-z) & =-R\left[p_{-n, 1}^{\prime}(R z)-p_{01}^{\prime}(R z)\right], \quad \forall n \in \mathbf{Z}, \\
p_{n k}(-z) & =(-1)^{k} R p_{-n, k}^{\prime}(R z), \quad \forall n \in \mathbf{Z}, \quad k \geq 2,
\end{aligned}
$$




$$
\begin{aligned}
\alpha_{n}(-z) & =-R \alpha_{-n}^{\prime}(R z), \quad \forall n \in \mathbf{Z}, \\
\gamma_{k l n}(-z) & =R \gamma_{-k,-l,-n}^{\prime}(R z)|C|^{-2}, \quad \forall k, l, n \in \mathbf{Z}, \\
g_{k l n}(-z) & =-R g_{-k,-l,-n}^{\prime}(R z)|C|^{-2}, \quad \forall k, l, n \in \mathbf{Z},
\end{aligned}
$$

$\forall z \in[0, L / R], C \neq 0$ being a constant, and the envelope functions are related as $A_{n}(-z, t)=C A_{-n}^{\prime *}(R z, t)$, $\forall n \in \mathbf{Z}$. Physically, it says that the two fiber lines compensate each other for dispersion and nonlinearity. Optical signals $A_{n}(-L / R, t), n \in \mathbf{Z}$, entering the first fiber line may be dispersed and nonlinearly distorted to become $A_{n}(0, t), n \in \mathbf{Z}$, which are converted into $A_{n}^{\prime}(0, t)=A_{-n}^{*}(0, t) / C^{*}, n \in \mathbf{Z}$, by the OPC. The second fiber line will then propagate the optical signals in a reversed manner with respect to the first. The final outputs signals $A_{n}^{\prime}(L, t)=A_{-n}^{*}(-L / R, t) / C^{*}, n \in \mathbf{Z}$, are exact replicas of the initial signals up to complex conjugation. It is noted that parts of one fiber line would amplify light in correspondence to the attenuation in parts of the other, and vice versa. A specialty fiber may be chosen with parameters satisfying equations $(38,39,41,42)$ to be the scaled mirror image of a transmission fiber which usually attenuates light. At the same time, erbium doping or Raman pumping should be employed to obtain the proper gain specified by equation (40).

A link with translational symmetry could be constructed to cancel weak nonlinearities up to the first order perturbation. Consider two fiber lines with opposite Kerr and Raman nonlinear coefficients but identical linear parameters. If (36) with $z \in[-L, 0]$ describe the signal propagation in one fiber line, then the signal dynamics in the other would be governed by similar equations with negative $\gamma$ and $g$ coefficients,

$$
\begin{array}{r}
\frac{\partial B_{n}}{\partial z}-i \mathrm{D}_{n}(z-L) B_{n}+\frac{\alpha_{n}(z-L)}{2} B_{n}=-i \sum_{k} \sum_{l} \gamma_{k l n}(z-L) B_{k} B_{l} B_{m}^{*} \exp \left[i \theta_{k l n}(z-L)\right] \\
+\sum_{k} \sum_{l} g_{k l n}(z-L) B_{k} B_{l} B_{m}^{*} \exp \left[i \theta_{k l n}(z-L)\right], \quad \forall n \in \mathbf{Z}, \quad 0 \leq z \leq L,
\end{array}
$$

which take the input $B_{n}(0, t), n \in \mathbf{Z}$ and give the output $B_{n}(L, t), n \in \mathbf{Z}$. When the signal intensity is not very high, so that the nonlinearity is weak and treated with perturbation theory, the output from each fiber line is a linearly dispersed version of the input, plus nonlinear distortions expanded in power series of the $\gamma$ and $g$ coefficients. By neglecting the higher order powers and keeping only the terms linear in $\gamma$ or $g$, it can be seen that the two fiber lines induce opposite nonlinear distortions to otherwise the same, linearly dispersed signals. If the overall dispersion of each line is compensated to zero and the signal loss is made up by a linear optical amplifier, then the two lines in cascade would comprise a transmission line with fiber nonlinearity annihilated up to the first order perturbation. The problem is that an optical fiber with negative nonlinear coefficients does not exist naturally. Fortunately, it can be simulated by a regular fiber with the help of OPC. Take a regular fiber with parameters $\left(\beta^{\prime}, \alpha^{\prime}, \gamma^{\prime}, g^{\prime}\right)$ that satisfy,

$$
\begin{aligned}
p_{n 1}^{\prime}(z)-p_{01}^{\prime}(z) & =R\left[p_{-n, 1}(R z-L)-p_{01}(R z-L)\right], \quad \forall n \in \mathbf{Z}, \\
p_{n k}^{\prime}(z) & =(-1)^{k-1} R p_{-n, k}(R z-L), \quad \forall n \in \mathbf{Z}, \quad k \geq 2, \\
\alpha_{n}^{\prime}(z) & =R \alpha_{-n}(R z-L), \quad \forall n \in \mathbf{Z}, \\
\gamma_{k l n}^{\prime}(z) & =R \gamma_{-k,-l,-n}(R z-L)|C|^{-2}, \quad \forall k, l, n \in \mathbf{Z}, \\
g_{k l n}^{\prime}(z) & =-R g_{-k,-l,-n}(R z-L)|C|^{-2}, \quad \forall k, l, n \in \mathbf{Z},
\end{aligned}
$$

$\forall z \in[0, L / R]$. The signal propagation in the regular fiber is then governed by,

$$
\begin{aligned}
\frac{\partial B_{n}^{\prime}}{\partial z}- & i \mathrm{D}_{n}^{\prime}(z) B_{n}^{\prime}+\frac{\alpha_{n}^{\prime}(z)}{2} B_{n}^{\prime}=i \sum_{k} \sum_{l} \gamma_{k l n}^{\prime}(z) B_{k}^{\prime} B_{l}^{\prime} B_{m}^{\prime *} \exp \left[i \theta_{k l n}^{\prime}(z)\right] \\
& -\sum_{k} \sum_{l} g_{k l n}^{\prime}(z) B_{k}^{\prime} B_{l}^{\prime} B_{m}^{\prime *} \exp \left[i \theta_{k l n}^{\prime}(z)\right], \quad \forall n \in \mathbf{Z}, \quad 0 \leq z \leq L / R
\end{aligned}
$$

which are solved by $B_{n}^{\prime}(z, t)=C B_{-n}^{*}(R z, t), n \in \mathbf{Z}$, and turn the input $B_{n}^{\prime}(0, t)=C B_{-n}^{*}(0, t), n \in \mathbf{Z}$, into the output $B_{n}^{\prime}(L / R, t)=C B_{-n}^{*}(L, t), n \in \mathbf{Z}$. The regular fiber equipped with OPCs at its two ends takes the input $B_{n}(0, t), n \in \mathbf{Z}$ and gives the output $B_{n}(L, t), n \in \mathbf{Z}$. In other words, the combination of a regular fiber and 
OPCs fulfils the function of the fictitious fiber with negative nonlinearity. The OPC at the output end of the regular fiber may be omitted in practice, as most applications would not differentiate between a signal and its conjugate.

It is interesting to compare the two methods of nonlinearity compensation. While a translational setup cancels weak nonlinearities only up to the first-order perturbation, a mirror configuration could fully compensate nonlinearities beyond perturbation. Between two fiber segments compensating each other, the translational symmetry requires opposite signs for the dispersion and the same sign for the gain/loss coefficient, and the mirror symmetry does the contrary. It is noted that each fiber line on one side of the OPC is not necessarily one fiber span, and the signal intensity does not have to evolve monotonically either. Both methods work fine when each side of the OPC consists of multiple fiber spans with optical amplifiers boosting the signal power, although the added noise makes perfect nonlinearity compensation impossible.

\section{SIMULATIONS}

Using a commercial software, computer simulations have been carried out to test the proposed methods of nonlinearity compensation. For a mirror setup, as shown on the left of Fig.1, the simulated link consists of a specialty fiber, an OPC, and a transmission fiber that is of the negative nonzero dispersion-shifted type, $200 \mathrm{~km}$ long, with loss coefficient $\alpha=0.2 \mathrm{~dB} / \mathrm{km}$, dispersion $D=-8 \mathrm{ps} / \mathrm{nm} / \mathrm{km}$, dispersion slope $S=0.08 \mathrm{ps} / \mathrm{nm}^{2} / \mathrm{km}$, effective mode area $A_{\text {eff }}=50 \mu \mathrm{m}^{2}$, Kerr and Raman coefficients that are typical of silica glass. The specialty fiber is a dispersion compensating fiber of the same material, but with parameters $\left(\alpha^{\prime}, D^{\prime}, S^{\prime}\right)=20 \times(-\alpha, D,-S)$ and $A_{\text {eff }}^{\prime}=12.5 \mu \mathrm{m}^{2}$. The nonlinearity of the specialty fiber can be switched on and off. ASE noise is added at the two ends of the link. The input consists of four WDM channels at $100 \mathrm{GHz}$ spacing, co-polarized, all RZ modulated at $10 \mathrm{~Gb} / \mathrm{s}$ with $33 \%$ duty. The power of all optical pulses is peaked at $100 \mathrm{~mW}$ when entering the transmission fiber. Fig.2 shows the received signals without and with nonlinearity in the specialty fiber respectively. The eye diagram on the left shows the overwhelming nonlinear distortion in the received signal, when the specialty fiber has no nonlinearity, but only pre-compensates the dispersion of the transmission fiber. When the nonlinearity of the specity fiber is turned on, the eye diagram on the right shows no nonlinear degradation, but only the effect of ASE noise, which demonstrates clearly the compensation of optical nonlinearity.
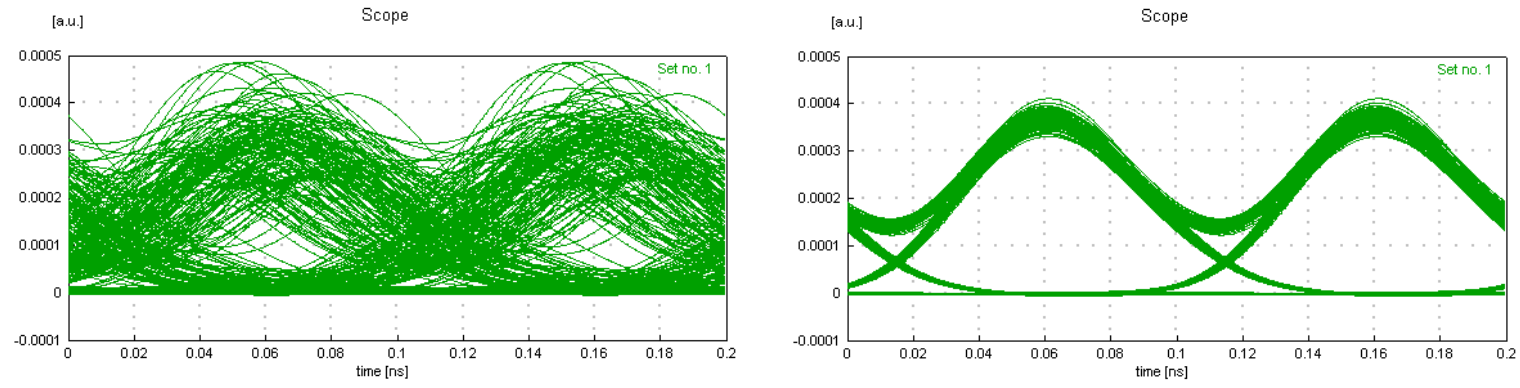

Figure 2. Received signals at the end of a simulated link. Left: only the dispersion of the transmission fiber is precompensated by the specialty fiber. Right: both dispersion and nonlinearity are pre-compensated.

The test system in translational symmetry is constructed with five 100-km spans on each side of the OPC to reach a total transmission distance of $1000 \mathrm{~km}$. On the left side of the OPC, each span consists of $100 \mathrm{~km}$ transmission fiber with parameters $\alpha=0.2 \mathrm{~dB} / \mathrm{km}, D=16 \mathrm{ps} / \mathrm{nm} / \mathrm{km}, S=0.055 \mathrm{ps} / \mathrm{nm}^{2} / \mathrm{km}$, and $A_{\text {eff }}=80$ $\mu \mathrm{m}^{2}$, then a stage of EDFA with $20 \mathrm{~dB}$ gain and $5 \mathrm{~dB}$ noise figure, followed by $20 \mathrm{~km}$ DCF with parameters $\left(5 \alpha,-5 D,-5 S, A_{\mathrm{eff}} / 5\right)$, and another stage of EDFA, again $20 \mathrm{~dB}$ gain, but negligible noise. In practice, the two stages may belong to just one EDFA. Both the transmission fiber and the DCF have the same nonlinear index $n_{2}=2.6 \times 10^{-20} \mathrm{~m}^{2} / \mathrm{W}$. On the right side of the OPC, the same fibers and EDFAs are used, but the DCF is placed before the transmission fiber in each span. This is to make the two sides translationally symmetric about the OPC, approximately, not exactly, because the dispersion slopes of the transmission fiber and the DCF do not satisfy the scaling rules. So the success of such setup would demonstrate some tolerance to the scaling rules. The 
input are four $40 \mathrm{~Gb} / \mathrm{s}$ WDM channels spaced by $200 \mathrm{GHz}$, each one is RZ-modulated with $33 \%$ duty cycle. The power of the optical peaks at $20 \mathrm{~mW}$. The left eye diagram of Fig. 3 displays the signal detected in the middle of the link, just before the OPC, and the right eye diagram shows the signal received at the end of the link. Clearly, the accumulated nonlinearity has already distorted the signal by the middle of the link. However, with the help of the OPC, the fiber spans in the rest of the link are able to undo the distortion caused by the previous spans. We have simulated two more cases for comparison, to further confirm the effect of nonlinearity compensation by the use of OPC and the translational symmetry. In one case, the same transmission link is used except that the OPC is removed. The received signal is shown on the left of Fig.4. In the other case, the OPC is still on, but the fiber spans on the right side of the OPC are identical to those on the left side. In particular, all spans have the transmission fiber placed before the DCF. The right diagram of Fig.4 shows almost no suppression of nonlinearity.
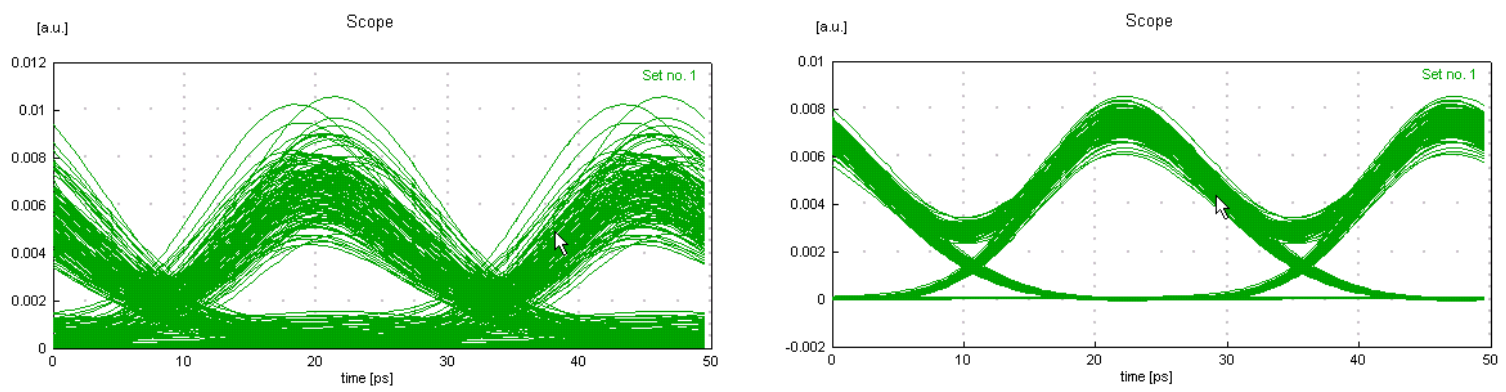

Figure 3. Received signals at the middle (left) and the end (right) of a simulated link.
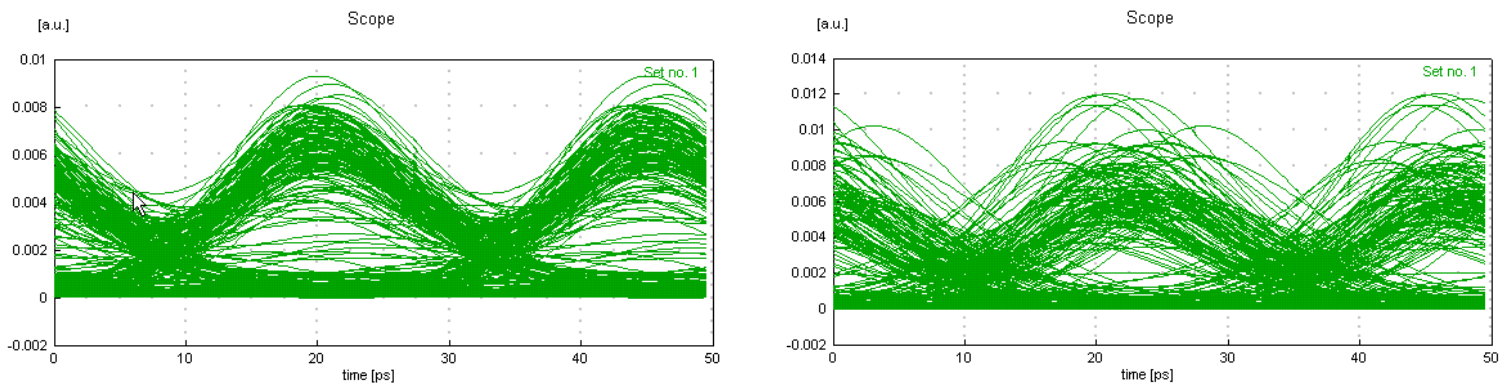

Figure 4. Received signals at the end of the link, with either the OPC removed (left), or the spans on the two sides of the OPC are identical (right).

\section{REFERENCES}

1. F. Forghieri, R. W. Tkach, and A. R. Chraplyvy, "Fiber nonlinearities and their impact on transmission systems," in Optical Fiber Telecommunications III A, I. P. Kaminow and T. L. Koch, eds. Academic Press, San Diego, 1997.

2. P. P. Mitra and J. B. Stark, "Nonlinear limits to the information capacity of optical fiber communications," Nature, vol. 411, pp. 1027 - 1030, June 2001.

3. Y. R. Shen, The Principles of Nonlinear Optics. John Wiley \& Sons, New York, 1984.

4. G. P. Agrawal, Nonlinear Fiber Optics, 2nd ed. Academic Press, San Diego, 1995.

5. Q. L. N.T., T. Veng, and L. Gruner-Nielsen, "New dispersion compensating module for compensation of dispersion and dispersion slope of non-zero dispersion fibres in the C-band," OFC'01, paper TuH5, Anaheim, CA, March 17 - 22, 2001. 
6. V. Srikant, "Broadband dispersion and dispersion slope compensation in high bit rate and ultra long haul systems," OFC'01, paper TuH1, Anaheim, CA, March 17 - 22, 2001.

7. M. Hirano, A. Tada, T. Kato, M. Onishi, Y. Makio, M. Nishimura, "Dispersion compensating fiber over 140nm bandwidth," ECOC'01, paper Th.M.1.4, Amsterdam, The Netherlands, September 30 - October 4, 2001.

8. L. Gruner-Nielsen, Y. Qian, B. Palsdottir, Y. Qian, P. B. Gaarde, S. Dyrbo, T. Veng, R. Boncek, and R. Lingle, "Module for simultaneous $\mathrm{C}+\mathrm{L}-$ band dispersion compensation and Raman amplification," OFC'02, paper TuJ6, Anaheim, CA, March 17 - 22, 2002.

9. K. Mukasa, H. Moridaira, T. Yagi, and K. Kokura, "New type of dispersion management transmission line with MDFSD for long-haul $40 \mathrm{~Gb} / \mathrm{s}$ transmission," OFC'02, paper ThGG2, Anaheim, CA, March 17 - 22, 2002.

10. M. Wandel, P. Kristensen, T. Veng, Y. Qian, Q. Le, L. Gruner-Nielsen, "Dispersion compensating fibers for non-zero dispersion fibers," OFC'02, paper WU1, Anaheim, CA, March 17 - 22, 2002.

11. M. Born and E. Wolf, Principles of Optics: Electromagnetic Theory of Propagation, Interference and Diffraction of Light, 7th ed. Cambridge University Press, 1999.

12. A. W. Snyder and J. D. Love, Optical Waveguide Theory. Chapman and Hall, Now York, 1983.

13. D. M. Pepper and A. Yariv, "Compensation for phase distortions in nonlinear media by phase conjugation," Opt. Lett., vol. 5, pp. 59 - 60, 1980.

14. S. Watanabe, G. Ishikawa, T. Naito, and T. Chikama, "Generation of optical phase-conjugate waves and compensation for pulse shape distortion in a single-mode fiber," J. Lightwave Technol., vol. 12, no. 12, pp. 2139 - 2146, 1994.

15. S. Watanabe and M. Shirasaki, "Exact compensation for both chromatic dispersion and Kerr effect in a transmission fiber using optical phase conjugation," J. Lightwave Techn., vol. 14, no. 3, pp. 243 - 248, 1996.

16. A. G. Grandpierre, D. N. Christodoulides, and J. Toulouse, "Theory of stimulated Raman scattering cancellation in wavelength-division-multiplexed systems via spectral inversion," IEEE Photon. Technol. Lett., vol. 11, no. 10, pp. 1271 - 1273, 1999.

17. I. Brener, B. Mikkelsen, K. Rottwitt, W. Burkett, G. Raybon, J. B. Stark, K. Parameswaran, M. H. Chou, M. M. Fejer, E. E. Chaban, R. Harel, D. L. Philen, and S. Kosinski, "Cancellation of all Kerr nonlinearities in long fiber spans using a $\mathrm{LiNbO}_{3}$ phase conjugator and Raman amplification," OFC'00, post-deadline paper, PD33, Baltimore, Maryland, 2000.

18. H. Yu and K.-P. Ho, "Limitation of stimulated Raman scattering cancellation in WDM systems via spectral inversion," IEEE Photon. Technol. Lett., vol. 12, no. 8, pp. 998 - 1000, 2000. 